\title{
A Transient but Protracted Geomagnetic Anomaly in the Sudbury Basin Following Two Near-Contiguous Intense Geomagnetic Storms
}

\author{
Michael A. Persinger, Blake T. Dotta \\ Laurentian University, Sudbury, Ontario, Canada \\ E-mail: mpersinger@laurentian.ca \\ Received March 28, 2011; revised June 7, 2011; accepted July 9, 2011
}

\begin{abstract}
During the maintained quiescence between solar cycle 23 and 24, two unusually intense (K-indices $=7$ ) global geomagnetic disturbances separated by 6 days occurred. They were followed by a protracted increase of between 150 and $200 \mathrm{nT}$ in the vertical component of our local magnetic field (Sudbury, Ontario). The duration of the variation anomaly was unusually long, about 3 weeks, before returning to baseline following a one week period of below average intensity characterized by approximately $50 \mathrm{~min}$ periodicities. We suggest this anomaly supports previous research that specific temporal patterns of increased global geomagnetic activity when matched with local impedance/reluctance of ore bodies created the condition for remarkable transient changes in the surface static intensity of magnetic fields.
\end{abstract}

Keywords: Anomalous Transients, Sudbury Basin, Magnetic Storm, Periodicity

\section{Introduction}

Arrays of magnetometers have been employed to discern geomagnetic variation anomalies [1]. They occur along continental edges, within specific crustal compositions, and over subsurface ore bodies. Although static magnetic configurations are associated with mineral deposits, such as nickel and magnetite [2] there are also additional vertical and horizontal transients usually lasting for hours to days during geomagnetic variations. The seminal work by Porath and Dziewosnki [3] reported that the anomalies corresponded to peaks in the energy of geomagnetic variation and showed a strong dependence of its periodicity, in the order of about $50 \mathrm{~min}$.

We have been collecting N-S, E-W and vertical (z) geomagnetic data once per min $24 \mathrm{hr} /$ day for more than 10 years with a MEDA FVM 400 vector magnetometer. The sensor for our instrument is positioned in the basement of a building of the university which is built beside the edge of the Sudbury Basin containing its large nickel deposit. During the first two weeks of April 2010 we measured an unprecedented transient geomagnetic anomaly. It was maintained for about three weeks and followed the strongest and temporally contiguous series of geomagnetic storms during the last two years. The period was also significant because there had been no mining or blasting, as verified by our RV-301 Helicorder seismographic unit, for more than 6 months because of a labour dispute.

\section{Methods and Materials}

The magnetometer was located in our basement laboratory. X (N-S), Y (E-W) and Z (vertical) measurements have been recorded every min to a Laptop computer for the last three years in that specific locality as a component of our research with $\mathrm{nT}$ range, $\mathrm{mHz}$ variations in background photon densities as measured by a photon multiplier tube. Geomagnetic information was obtained by e-mail from SWPC Production Subscription service from NOAA Space Weather. For the present analyses the median values for the 1,440 measurements for the $\mathrm{X}, \mathrm{Y}$, and $\mathrm{Z}$ directions per day were obtained for serial daily comparisons. We employ median values to minimize the effects of occasional cultural effects. Because the greatest changes occurred in the vertical axis $(Z)$, these data were selected to be presented.

\section{Measurements}

As can be seen in Figure 1, a series of typical perturba- 
tions began within the vertical component of the field after the sudden impulse (S) on 2 April. However on 13 April the vertical intensity increased by between 150 and $200 \mathrm{nT}$ and remained elevated until 3 May. Following this maintained peak the value dropped below baseline levels (indicated by "a" in Figure 1) for about a week before returning to the typical local values. Figure 2 shows the approximately 50 min periodicity with peakto-peak amplitude of about $30 \mathrm{nT}$ that occurred during this interval.

Considering the importance of periodicity and largescale impedance/inductance factors for the creation of transient variation anomalies $[1,2]$, the actual sequence of geomagnetic events were considered important. The approximate times for the $\mathrm{K}$ events $>4$ are shown as vertical dotted lines in Figure 1. The specific date and

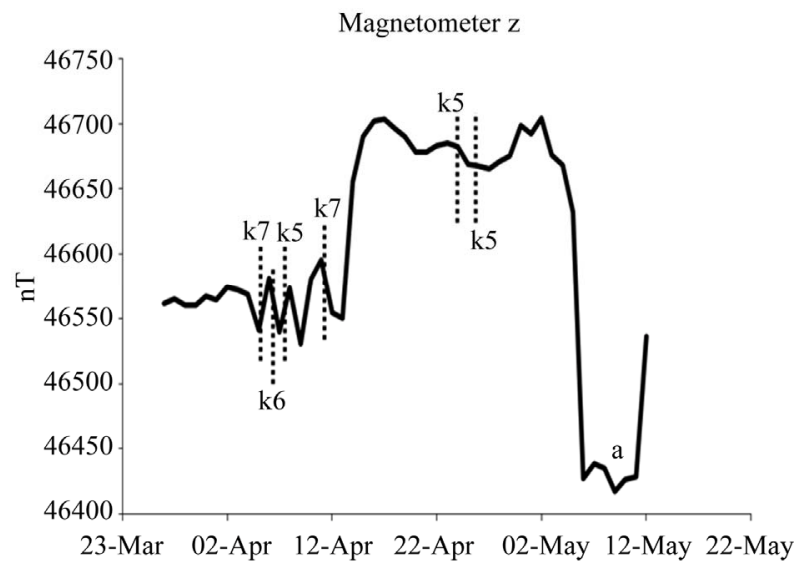

Figure 1. Potential geomagnetic transient enhancement of the vertical $(Z)$ component of the geomagnetic field following a specific sequence of geomagnetic storms (indicated by vertical dotted lines) whose intensities are indicated by k-values. The net protracted increase was about $150 \mathrm{nT}$.

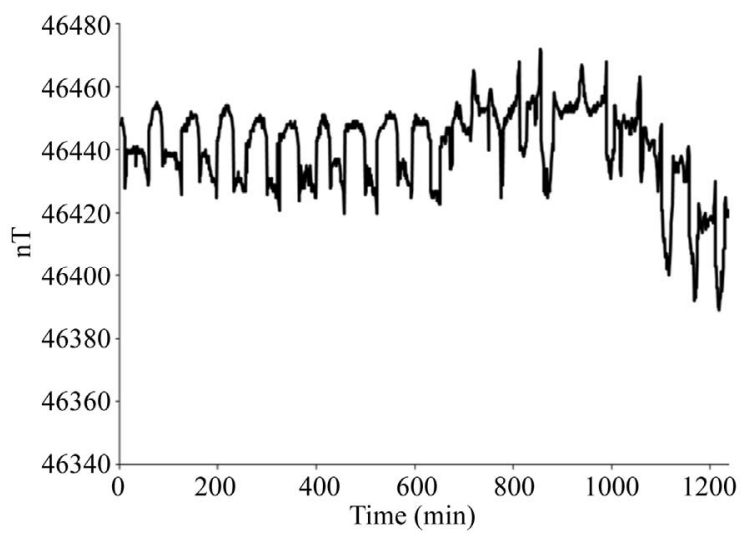

Figure 2. Sample periodicity (about $50 \mathrm{~min}$ ) that occurred during the "undershoot" component (indicated by "a" in Figure 1) of the potential geomagnetic transient shown in Figure 1. This periodicity was no longer evident when the intensity returned to baseline levels. time of the events, according to alerts $(\mathrm{K}>3)$ from Boulder, were: 2 April, GSI $19 \mathrm{nT}$ (0721 hr) 5 April, GSI $38 \mathrm{nT}(0826 \mathrm{hr}) ; 5$ April, K-5 (0916 hr); 5 April, K-7 (0955 hr), 6 April, K-6 (0422 hr), 7 April, K-5 (0847 hr), 11 April, K-5 (0224 hr), K-6 (0225 hr), K-7 (0240 hr); 22 April, K-4 (0058 hr).

\section{Calculations and Assumptions}

The Sudbury Basin, due to an impact of a meteor of about $10 \mathrm{~km}$ diameter about 1.8 billion years ago, is 62 $\mathrm{km} \times 30 \mathrm{~km} \times 15 \mathrm{~km}$ (deep). This constitutes an area of $1.8 \times 10^{9} \mathrm{~m}^{2}$. The electric field induced in a space is $\mathrm{V}=\partial \mathrm{B} / \partial \mathrm{t} \cdot \mathrm{m}^{2}$. Assuming the typical variation of about 100 $\mathrm{s}$ in periodicity and a field change in the order of $500 \mathrm{nT}$ (from the storm), the result would be: $\left(5 \times 10^{-7} \mathrm{~T} / 10^{2}\right.$ s) $1.8 \times 10^{9} \mathrm{~m}^{2}$ or $\sim 10 \mathrm{~V}$. The first order current, inferred by V/Ohm-m and with an average of $\sim 10^{2} \mathrm{Ohm}-\mathrm{m}$ for deposits, would be $\sim 10^{-1} \mathrm{~A} / \mathrm{m}$. When multiplied by the length of the basin there would be a potential $\sim 5.6 \times 10^{3}$ A. The magnetic field strength from a current is $\mathrm{B}=$ $\mu \mathrm{i} / 2 \pi \mathrm{r}$. Hence $\left(1.26 \times 10^{-6} \mathrm{~N} / \mathrm{A}^{2} \cdot 5.6 \times 10^{3} \mathrm{~A}\right)$ divided by $6.28 \cdot 1.0 \times 10^{4} \mathrm{~m}$ (the estimated distance of the laboratory from the conductive range of the ore body), is 1.1 and $10^{-7} \mathrm{~T}$ or $110 \mathrm{nT}$. This is within the range observed even with minimally rigorous assumptions.

\section{Discussion and Implications}

Our measurements suggest that following a particular temporal pattern of enhanced global geomagnetic activity, an anomalous increase in of between 150 and $200 \mathrm{nT}$ occurred within the vertical component was maintained for about 3 weeks. However, most variation anomalies' durations have been in the order of days. We suggest that

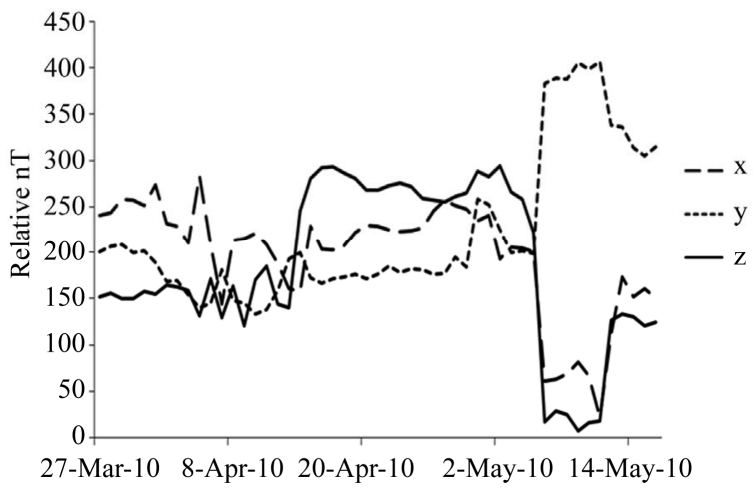

Figure 3. Superimposed intensities of the north-south $(X)$, east-west $(Y)$ and vertical $(Z)$ components of the local geomagnetic field during the transient anomaly. During the periodicities associated with the undershoot (Figure 2), the magnetic field increase was displayed within the E-W direction. 
this specific temporal sequence of geomagnetic storms may have maintained the current to produce this anomaly. During this period there was also no mining (or blasting). Another low probability possibility is that there was undetected homogeneous strain within the basin that allowed a minimum dissipation of current. Strain accumulation during sudden and prolonged termination of blasting in some areas might be associated with in areas with increased strain.

Local cultural artifacts from, for example machinery, were not responsible for several reasons. First the elevated magnitude was more or less consistent $24 \mathrm{hr}$ per day for the three week period. Second, the one week undershoot was associated with a periodicity that is remarkably similar values in the vicinity of $50 \mathrm{~min}$ that are responsible for such anomalous fields in other ore-bearing regions [3]. Within the Great Plains Anomaly during various geomagnetic "storms" the various periods ranged between $24 \mathrm{~min}$ and $85 \mathrm{~min}$. In many ideal circuits the temporal characteristics associated with the "charging" is reflected during the period of "discharging". In response to queries by a referee for the manuscript the three-coordinates of the magnetometer data were superimposed.
As can be see in Figure 3 only the E-W component was markedly elevated during the periodicity of the "discharging". This component would have been effectively parallel to the W/SW to E/NE orientation of the central moment of the Basin. The fact that the marked increase in this component of the static magnetic component occurred only during the $\sim 50$ min periodicities and not during the main transient might be considered support for our explanation.

\section{References}

[1] D. J. Gough, "The Geophysical Significance of Geomagnetic Variation Anomalies," Physics of the Earth and Planetary Interiors, Vol. 7, No. 3, 1983, pp. 379-388. doi:10.1016/0031-9201(73)90062-9

[2] P. J. Gunn, M. C. Smith, "Magnetic Responses Associated with Mineral Deposits," AGSO Journal of Australian Geology \& Geophysics, Vol. 17, 1997, pp. 145-158.

[3] H. Porath, A. Dziewonski, "Crustral Resistivity Anomalies from Geomagnetic Deep-Sounding Studies," Reviews of Geophysics and Space Physics, Vol. 9, No. 4, 1971, pp. 891-915. doi:10.1029/RG009i004p00891 